\title{
Dificuldades no processo de aprendizagem de Algoritmos: uma análise dos resultados na disciplina de AL 1 do Curso de Sistemas de Informação da FAETERJ - Campus Paracambi
}

\section{Difficulties in Algorithms learning process: an analysis of results in AL 1 discipline at Course of Information Systems in the FAETERJ - Campus Paracambi}

\author{
1 José Augusto Teixeira de Lima Junior \\ ${ }^{2}$ Carlos Eduardo Costa Vieira \\ ${ }^{3}$ Priscila de Paula Vieira
}

\footnotetext{
1 (Sistemas de Informação - FAETERJ/USS) - junior.jaugusto@gmail.com

2 (Sistemas de Informação - FAETERJ/UniFOA) - cadu.vieira@gmail.com

3 (Sistemas de Informação - FAETERJ) - priscilapaula.vieira@gmail.com
}

\section{Resumo}

Algoritmos podem ser definidos como uma sequência de ações executáveis para a obtenção de uma solução para um determinado tipo de problema. A disciplina inicial de Algoritmos, que é o primeiro contato dos discentes com a área de Programação de Computadores, é a base para qualquer curso de Computação. Porém, para muitos alunos, constitui uma barreira intransponível, pois apresenta conteúdos envolvendo interpretação de texto, raciocínio lógico e matemática. Este artigo tem como objetivo fazer uma análise das dificuldades apresentadas pelos alunos ao cursar a disciplina de Algoritmo e Linguagem de Programação 1 do curso de Sistemas de Informação, da Faculdade de Educação Tecnológica do Estado do Rio de Janeiro - Campus Paracambi. Para isso, foi feito um levantamento dos índices de aprovação/ reprovação na disciplina no triênio 2010-2012 e aplicado um questionário junto aos alunos sobre as dificuldades de assimilação dos conteúdos da disciplina. 0 estudo mapeou, por meio dos dados coletados, os pontos críticos que geram maiores problemas de aprendizagem, para que medidas corretivas possam ser direcionadas com o objetivo de melhorar o aprendizado dessa importante disciplina introdutória da área de Computação.

\section{Palavras-chave}

Algoritmos; ensino; aprendizagem; dificuldades.

\begin{abstract}
Algorithms can be defined as a sequence of executable actions to obtain a solution for many kind of problems. The initial discipline of algorithms, which is the first contact of students with Computer Programming area is the basis for any course of Computing. But for many students, is an insurmountable barrier, because it presents content involving reading comprehension, logical reasoning and mathematics. This article aims to analyze the difficulties presented by the students to take the disciplines of Algorithms and Programming Language 1 at course of Information Systems in the College of Technological Education of the State of Rio de Janeiro - Campus Paracambi. For this, a survey was made of the contents of pass / fail in the discipline in the 20102012 period and a questionnaire to the students about the difficulties of assimilation of the course content. The study was mapped through the collected data, the critical points that generate higher learning problems so that corrective measures could be directed in order to improve learning this introductory discipline so important for Computer Science area.
\end{abstract}

\section{Keywords}

Algorithms; teaching; learning; difficulties.

\section{Como você deve citar?}

LIMA JUNIOR, José Augusto Teixeira; VIEIRA, Carlos Eduardo Costa; VIEIRA, Priscila de Paula. Dificuldades no processo de aprendizagem de Algoritmos: uma análise dos resultados na disciplina de AL1 do Curso de Sistemas de Informação da FAETERJ - Campus Paracambi. Cadernos UniFOA, Volta Redonda, n. 27, p. 5-15, abr. 2015. 
Dificuldades no processo de aprendizagem de Algoritmos: uma análise dos resultados na disciplina de AL 1 do Curso de Sistemas de Informação da FAETERJ - Campus Paracambi

\section{INTRODUÇÃO}

Os cursos de Tecnologia ou Graduação na área de Computação apresentam a disciplina de Algoritmos como base conceitual inicial, imprescindível e obrigatória. As definições de algoritmos variam de autor para autor, mas seu fundamento principal nunca muda: receber entradas, processá-las e produzir saídas.

Segundo Forbellone e Eberspacher (2005, p. 3), "um algoritmo pode ser definido como uma sequência de passos que visam atingir um objetivo bem definido". Na visão de Cormen et al. (2002, p. 3 ), caracteriza-se como "qualquer procedimento computacional bem definido que toma algum valor ou conjunto de valores como entrada e produz algum valor ou conjunto de valores como saída". Para Medina e Fertig (2006, p. 13) é visto como "um procedimento passo a passo para a solução de um problema, ou ainda, como uma sequência detalhada de ações a serem executadas para realizar alguma tarefa". Carboni (2003, p. 12) o considera como "uma sequência de procedimentos finitos que, se forem executados em determinado período de tempo, chegará ao seu objetivo". Já Farrer et al. (1999, p. 14) descrevem algoritmo como "um conjunto de comandos que, obedecidos, resultam numa sucessão finita de ações".

É notório que em todos os cursos de tecnologia ou graduação da área de Computação, a disciplina de Algoritmos constitui eixo básico essencial na formação dos alunos. Por ser o primeiro contato direto dos discentes com ambientes que trabalham os conceitos de raciocínio lógico, matemática e programação, pode-se evidenciar que em todos os cursos, tanto de instituições públicas quanto privadas, a disciplina se faz presente desde o primeiro ano da formação do aluno.

Segundo a Sociedade Brasileira de Computação (2003), a estrutura curricular básica dos cursos de Computação apresenta em sua composição uma área de formação básica denominada "Ciência da Computação", que é subdividida em três partes: programação, computação e algoritmos e arquitetura de computadores. Tal estruturação demonstra de forma clara a real importância que é direcionada aos algoritmos no currículo de formação discente na área em foco.

Dada a devida importância dessa disciplina, observa-se de forma bastante significativa que ela constitui grande "divisor de águas" nos cursos de Computação, pois causa grande impacto no primeiro ano de formação, evidenciando de forma indireta, para quem não consegue compreender o assunto, uma grande barreira que impede a progressão do discente para outros períodos e não somente isso, 0 entendimento de disciplinas de programação que também fazem parte do currículo.

Um ponto importante a ser observado é que nem todos os alunos possuem o mesmo nível de conhecimentos, tendo em vista que as formações do ensino médio são distintas de um aluno para outro, sem contar com o tempo que muitos passam sem estudar até seu ingresso na faculdade. Outra situação crítica encontrada seria o reconhecimento dos procedimentos necessários para se chegar à solução do algoritmo, ou seja, os alunos têm problemas em identificar os dados, o resultado esperado e o que é preciso para resolver o problema.

O objetivo do artigo é levantar as dificuldades dos alunos no processo de aprendizagem de algoritmos dentro de suas estruturas básicas (fundamentos iniciais, estruturas sequencial, condicional e repetição, vetores e matrizes) na disciplina de Algoritmo e Linguagem de Programação 1 (AL1) do Curso Superior de Tecnologia em Sistemas de Informação (CSTSI) da Faculdade de Educação Tecnológica do Estado do Rio de Janeiro (FAETERJ-Campus Paracambi), com o objetivo de entender e melhorar o processo de aprendizagem de algoritmos. 
Primeiramente, foi realizada uma pesquisa bibliográfıca utilizando materiais formalmente publicados sobre o assunto como livros, artigos, trabalhos de conclusão de curso e publicações em sites confiáveis. Procurou-se bibliografias que abordassem a definição de algoritmos, o processo de aprendizagem, suas unidades básicas, desde os conceitos iniciais de variáveis, constantes, operadores até as estruturas de dados homogêneas (vetores e matrizes). Posteriormente, foi realizado um levantamento dos índices de aprovação e reprovação na disciplina de AL1 no triênio letivo (2010/2011/2012). Por último, procurou-se mapear os maiores pontos de dificuldade por meio de uma pesquisa de campo com a utilização de um questionário aplicado aos alunos.

Este artigo está organizado como se segue: a Seção 2 apresentará o processo de aprendizagem de Algoritmos; a Seção 3 descreverá as Unidades de Aprendizagem utilizadas na disciplina de AL1; a Seção 4 mostrará a análise do processo de aprendizagem de algoritmos no CSTSI da FAETERJ-Campus Paracambi e, por último, a Seção 5 apresentará as Conclusões e Propostas de Trabalhos Futuros.

\section{PROCESSO DE APRENDIZAGEM DE ALGORITMOS}

A educação engloba os processos de ensinar e aprender, possibilitando a capacidade de agir intelectualmente e pensar produtivamente. Ensinar é passar conhecimento e vinculado ao ensino está o aprender que se conceitua em absorver a informação. Esses métodos têm sido analisados por diversos autores a partir de seus princípios e suas respectivas teorias e abordagens com o objetivo de explicar o processo em estudo.

Alguns direcionamentos devem ser levados em consideração, quando se pensa em processo de aprendizagem de algoritmos.

Podem ser elencados a motivação, entendida como o conjunto de causas que incentivam um indivíduo a realizar determinadas ações até alcançar seus objetivos. Para Balancho e Coelho (1996 apud Moraes e Varela, 2007) é um processo, e como tal, é aquilo que suscita ou incita uma conduta, que sustenta uma atividade progressiva, que canaliza essa atividade para um dado sentido. Pensando em algoritmos, muitos alunos que são aprovados no processo seletivo acadêmico desconhecem a disciplina, o que dificulta muito a abordagem inicial de conceitos. Tal afirmativa reforça a necessidade de se trabalhar de maneira motivacional com os alunos, para que os mesmos possam render na disciplina.

O segundo direcionamento seria a didática que, segundo Libâneo (1990 apud Pacievitch, 2009), é caracterizada como a "teoria do ensino" por investigar os fundamentos, as condições e as formas de realização do ensino. Uma vertente mais direta do conceito aponta a mesma como a "arte de ensinar" por meio de técnicas e métodos de ensino. Sendo assim, os conceitos de algoritmos para os alunos do primeiro ano dos cursos de Computação precisam ser trabalhados de maneira muito particular sob o ponto de vista da preocupação com a aprendizagem. Dar condições de aprendizado por meio dos fundamentos, agregados a ferramentas de produtividade (ambientes de programação) tornam mais completa a abordagem e possibilitam o exercício da didática de maneira mais abrangente.

O terceiro direcionamento é a metodologia, que está diretamente relacionada com a forma de ensino, ferramentas e recursos utilizados. A metodologia permite que o docente trabalhe com o aluno, dentro da disciplina de Algoritmos, a interpretação de textos, o raciocínio lógico e o desenvolvimento matemático. Segundo Koliver, Dorneles e Casa (2004 apud Ferreira, 2007), as disciplinas voltadas para o ensino de algoritmos são problemáticas, o que traduz alto índice de reprovações e desistência, sendo um dos principais motivos para tal, a falta de preparo dos alunos oriunda de sua formação no ensino fundamental e médio. 
Dificuldades no processo de aprendizagem de Algoritmos: uma análise dos resultados na disciplina de AL 1 do Curso de Sistemas de Informação da FAETERJ - Campus Paracambi

Por último, pode-se citar o raciocínio lógico, que é, sem dúvida, uma das maiores preocupações no que tange à disciplina de Algoritmos. Conforme já mencionado, a formação deficitária dos alunos, no que diz respeito a forma de pensar, amplia o universo de dificuldades encontradas, quando o mesmo necessita estruturar um determinado problema e apresentar, de forma sistematizada, a solução para ele. Segundo Raabe e Silva (2005 apud Jesus e Brito, 2009), atrelados à questão do raciocínio lógico, apontam-se de forma complementar, a capacidade de abstração, as dificuldades de interpretação de problemas e a base matemática que tornam ainda maior o nível de desmotivação e, por conseguinte, a desistência da disciplina e, posteriormente, do próprio curso.

\section{UNIDADES DE APRENDIZAGEM DA DISCIPLINA DE AL1}

A disciplina de Algoritmos pode ser estruturada no que se chama de Unidades de Aprendizagem. Tais unidades direcionam o conteúdo à maneira como os autores organizam os tópicos de aprendizado, procurando montar uma sequência lógica de conhecimentos até que se chegue a um nível esperado de conceitos a serem absorvidos pelos alunos. Pode-se evidenciar pelo referencial teórico que as unidades podem ser divididas em três grandes partes: tópicos fundamentais, estruturas de controle de fluxo dos algoritmos e estruturas de dados compostas homogêneas.

Nos tópicos fundamentais, são abordados os princípios básicos conceituais que servem de pilar de sustentação para o ensino de algoritmos. Nesse contexto, são trabalhados operadores aritméticos, relacionais e lógicos, tipos de dados, variáveis e constantes.

As estruturas de controle de fluxo podem ser divididas em três estruturas: sequencial, decisão ou condição e repetição. Segundo Forbellone e Eberspacher (2005) e Farrer et al. (1999), a estrutura sequencial apresenta um conjunto de ações primitivas que serão executadas em uma sequência linear de cima para baixo e da esquerda para a direita, isto é, na mesma ordem em que foram escritas.

A estrutura de decisão, seleção ou condição, para Forbellone e Eberspacher (2005) e Farrer et al. (1999), permite a escolha de um grupo de ações (bloco) a ser executado, quando determinadas condições, representadas por expressões lógicas ou relacionais, são ou não satisfeitas.

Finalizando, para Farrer et al. (1999), a estrutura de repetição permite que uma sequência de comandos seja executada repetidamente até que uma determinada condição de interrupção seja satisfeita. Segundo Forbellone e Eberspacher (2005), a estrutura de repetição pode ser diferenciada de acordo com o posicionamento dos testes de parada, que podem ser inseridos no início ou no fim da estrutura, implementados com comandos "enquanto" ou "repita", respectivamente, ou ainda, utilizandose de variável de controle de repetições (comando "para").

A terceira e última parte das Unidades de Aprendizagem apresenta conceitos de estruturas de dados homogêneas unidimensionais e bidimensionais. Na compreensão de Forbellone e Eberspacher (2005), assim como na teoria dos conjuntos, uma variável pode ser interpretada como um elemento e uma Estrutura de Dados, como um conjunto. Quando uma determinada Estrutura de Dados é composta de variáveis com o mesmo tipo primitivo de dados, temos um conjunto homogêneo de dados. Pode-se considerar que uma variável composta homogênea seja uma alcateia e seus elementos (variáveis) sejam como lobos (que são da mesma espécie). Para Farrer et al. (1999), variáveis compostas homogêneas correspondem à posição de memória, identificadas por um mesmo nome, individualizadas por índices e cujo conteúdo é do mesmo tipo. Por último, Medina e Fertig (2006) mostram que as variáveis compostas homogêneas também são conhecidas como vetores. Pode-se considerar tais variáveis como um subconjunto de um determinado tipo de dados. 
Segundo Farrer et al. (1999, p. 93), as estruturas homogêneas unidimensionais ou vetores são um "conjunto de dados referenciados por um mesmo nome e que necessitam de somente um índice para que seus elementos sejam endereçados são ditos compostos unidimensionais". Farrer et al. (1999, p. 110) definem ainda estrutura de dados homogênea bidimensional ou matriz como "um conjunto de dados referenciado por um mesmo nome e que necessita de mais de um índice para ter seus elementos individualizados, no caso dois índices e, por isso, é dito bidimensional."

\section{ANÁLISE DO PROCESSO DE APRENDIZAGEM DE ALGORITMOS NO CSTSI}

O trabalho fundamentou-se motivacionalmente por meio da observação da dificuldade de aprendizado de grande parte dos alunos matriculados na disciplina de AL1, ao longo dos anos. Foi separado para amostragem, o triênio 2010-2012.

A FAETERJ-Paracambi possui dois turnos de aulas, manhã e noite. Foram mapeadas informações sobre cursar ou não a disciplina após estar matriculado, índice de aprovação e reprovação direta, aprovação e reprovação com exame final e o fechamento final de aprovados e reprovados.

Após o levantamento da situação acadêmica, foi realizada uma pesquisa por meio de um questionário (Apêndice $A$ ), no qual os alunos puderam responder questões com ênfase em sua formação no ensino médio, o número de dependências (reprovações) em AL1, conhecimento prévio de algoritmos, dificuldade no entendimento e interpretação de problemas, dificuldade no entendimento de lógica de programação e as dificuldades de aprendizado, segundo as Unidades de Aprendizagem. Para essa última questão, foram apontados os seguintes tópicos: conceitos de algoritmos e sua utilidade; variáveis, constantes e tipos de dados; atribuição, operandos/operadores e expressões; entrada, processamento e saída; estrutura sequencial, estrutura condicional; estrutura de repetição; vetores e matrizes.

\subsection{Análise dos Resultados Acadêmicos no Triênio 2010-2012}

A primeira dimensão avaliada foi com relação ao índice de abandono da disciplina. Para o estudo, foram considerados os alunos regularmente matriculados na disciplina que puderam ser classificados como "cursantes", que cumpriram com suas atividades acadêmicas até o final do período letivo e os "não cursantes", caracterizados pelo índice de presença em poucas aulas do programa semestral da disciplina ou ainda aqueles que não realizaram nenhuma das avaliações que geram os índices para aprovação.

Foram levantados os dados de 663 alunos que se enquadravam no foco da pesquisa, dos quais, $77 \%$ (511) cursaram a disciplina até o final e 23\% (152) não cursaram ou abandonaram a mesma (Figura 1). 
Dificuldades no processo de aprendizagem de Algoritmos: uma análise dos resultados na disciplina de AL 1 do Curso de Sistemas de Informação da FAETERJ - Campus Paracambi

Figura 1 - Demonstrativo de Situação Acadêmica - Triênio 2010-2012

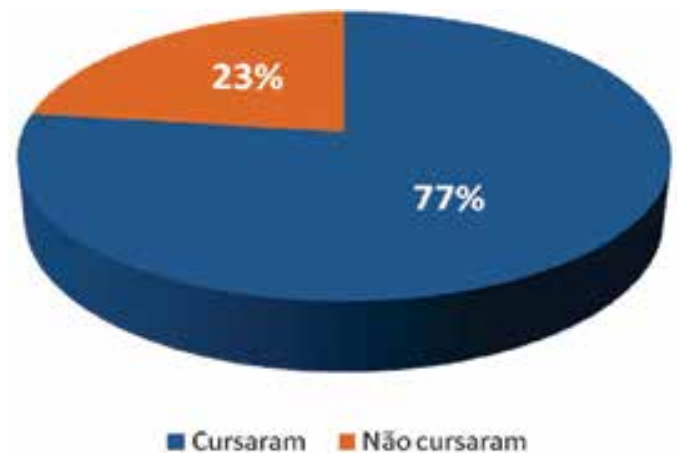

Fonte: dos autores, 2015.

A segunda dimensão avaliada foi o aproveitamento dos alunos na disciplina. Os alunos aprovados diretamente, que obtiveram média final igual ou superior a 7,0; os reprovados diretamente, que obtiveram média final inferior a 5,0, até o ano de 2012, e, inferior a 4,0, a partir do ano de 2012 e, por fim, os discentes que ficaram em avaliação final, avaliação extra aplicada aos alunos que obtiveram média final entre 5,0 e 7,0, até o ano de 2012 ou média final entre 4,0 e 7,0, a partir do ano de 2012.

No estudo, pôde-se observar que $30 \%$ dos alunos da amostra foram aprovados de forma direta na disciplina, $40 \%$ foram reprovados e o restante, que totalizou $30 \%$, foi direcionado para a avaliação final. Tais índices apontam uma quantidade significativa de reprovação face à aprovação primária, sem levar em consideração a oportunidade da avaliação extra (Figura 2).

Figura 2 - Demonstrativo de Aproveitamento - Triênio 2010-2012

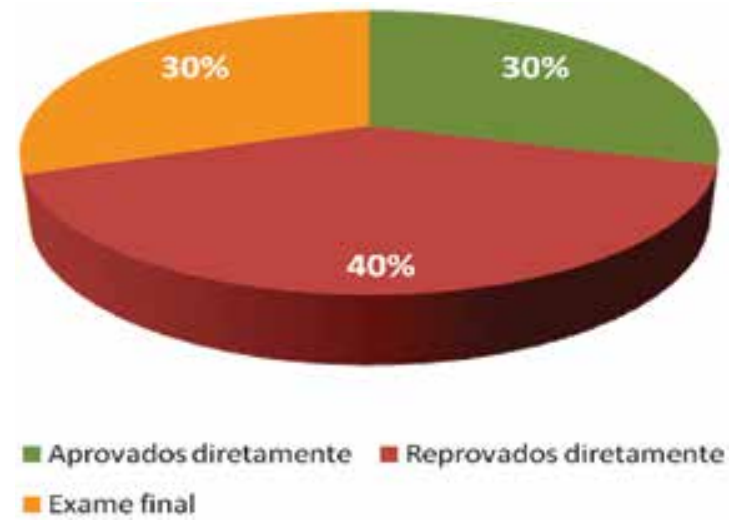

Fonte: dos autores, 2015.

A terceira dimensão levantada foi direcionada para os alunos que ficaram em exame final, em que foi observado o índice de $55 \%$ de aprovação e $45 \%$ de reprovação. Um fator relevante para essa dimensão é a média para aprovação no exame final que passa de 7,0 (aprovação direta) para 6,0. Nessa modalidade, ficou evidenciado um ligeiro aumento no percentual de aprovados que pode ser explicado em virtude da diminuição da nota final para a progressão na disciplina (Figura 3). 
Figura 3 - Demonstrativo de Aproveitamento no Exame Final - Triênio 2010-2012

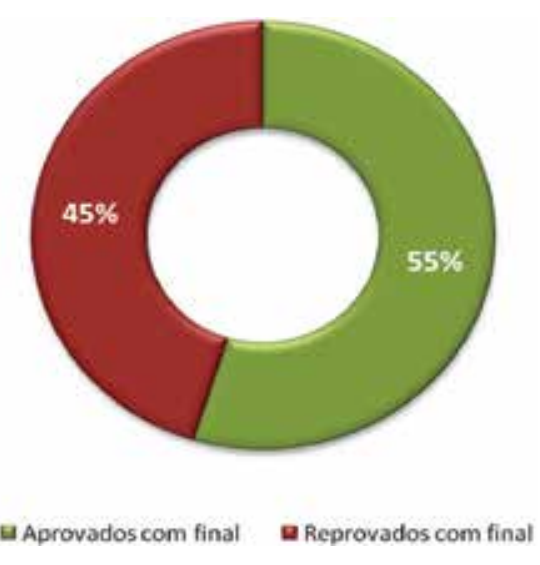

Fonte: dos autores, 2015.

A última dimensão, que consolida as demais, fechando a visão geral do aproveitamento acadêmico da disciplina de $A L 1$, é orientada para a aprovação e reprovação independente da forma (direta ou exame final). Os números apontam a motivação principal da pesquisa, visto que, para a situação da disciplina de AL1, há mais reprovação do que aprovação (Figura 4).

Figura 4 - Consolidação dos Resultados Aprovados/Reprovados - Triênio 2010 - 2012

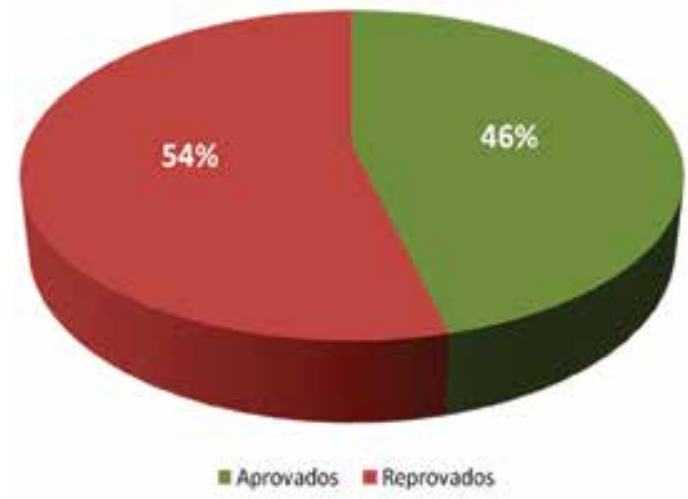

Fonte: dos autores, 2015

\subsection{Análise das Dificuldades de Aprendizado na Disciplina de AL1}

Com foco no objetivo da pesquisa, foi elaborado e aplicado um questionário junto aos alunos do curso de Tecnologia em Sistemas de Informação dos turnos da manhã e noite, para que os mesmos pudessem responder sobre suas dificuldades de assimilação de conteúdos dentro de cada Unidade de Aprendizagem.

A amostragem contou com 112 alunos participantes de um total de 308 , isentando os alunos de primeiro período, o que representa $36,4 \%$ do universo da amostra.

As perguntas do questionário foram planejadas para apontar possíveis indicadores que precisam ser melhorados, com relação à atenção docente, no que tange a abordagem de tais conteúdos. 
Dificuldades no processo de aprendizagem de Algoritmos: uma análise dos resultados na disciplina de AL 1 do Curso de Sistemas de Informação da FAETERJ - Campus Paracambi

A primeira que apresenta relevância para análise é a formação do ensino médio que, segundo as respostas, $60 \%$ são egressos do ensino regular, $20 \%$ do técnico em informática, $1 \%$ de formação de professores e $19 \%$ de outros cursos.

Com relação ao conhecimento prévio de lógica de programação, o resultado apontou que $71 \%$ dos alunos nunca tinham estudado tal conteúdo e $29 \%$ estudaram. 0 índice superior a $20 \%$ (apontado pelos alunos que cursaram técnico em Informática) pode ser justificado pelo fato de alguns alunos terem cursado, em paralelo à formação do ensino médio, cursos de Informática.

A dificuldade de entendimento dos problemas para o direcionamento das soluções apontou certo equilíbrio entre as respostas, conforme demonstra a Figura 5.

\section{Figura 5 - Dificuldades para o Entendimento dos Problemas}

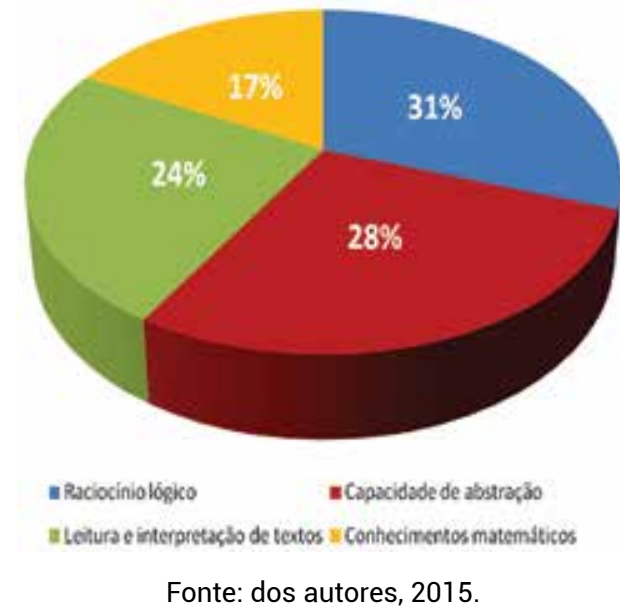

Com os dados, pôde-se observar que o raciocínio lógico foi o principal problema dos alunos, totalizando $31 \%$ das respostas; seguido da capacidade de abstração, com $28 \%$; leitura e interpretação de textos, com $24 \%$ e conhecimentos matemáticos, $17 \%$.

O ponto chave do artigo é o questionário, no qual o aluno responde sobre o conteúdo que maior causou dificuldade de aprendizado, no seu entender. Foram elencados os tópicos fundamentais do programa da disciplina, que englobam as Unidades de Aprendizagem.

A Figura 6 expressa a distribuição das respostas dessa questão, na qual os alunos puderam assinalar mais de uma alternativa, visto que o mesmo poderia sentir dificuldades em mais de uma abordagem.

As estruturas de dados homogêneas, matrizes e vetores foram os conteúdos que mais trouxeram dificuldades de aprendizado para os alunos, apresentando índices de $34 \%$ e $23 \%$, respectivamente, totalizando juntas $57 \%$. Em seguida, ficou evidenciado que as estruturas de repetição representaram $13 \%$; estruturas de decisão, $8 \%$; atribuições, operandos/operadores e expressões, $7 \%$; estruturas sequenciais, $6 \%$; variáveis, constantes e tipos de dados, $4 \%$; conceitos de algoritmos e sua utilidade, $4 \%$; e, por fim, o entendimento de entrada, processamento e saída, com $1 \%$.

Pela pesquisa realizada, a maior dificuldade inicia-se a partir da estrutura de repetição, na qual os alunos precisam visualizar e compreender que os códigos ou trechos de códigos podem estar dentro de estruturas que realizam repetições sucessivas, de acordo com as condições que precisam ser atendidas para seu término. 
Figura 6 - Conteúdos que apontaram Maiores Dificuldades

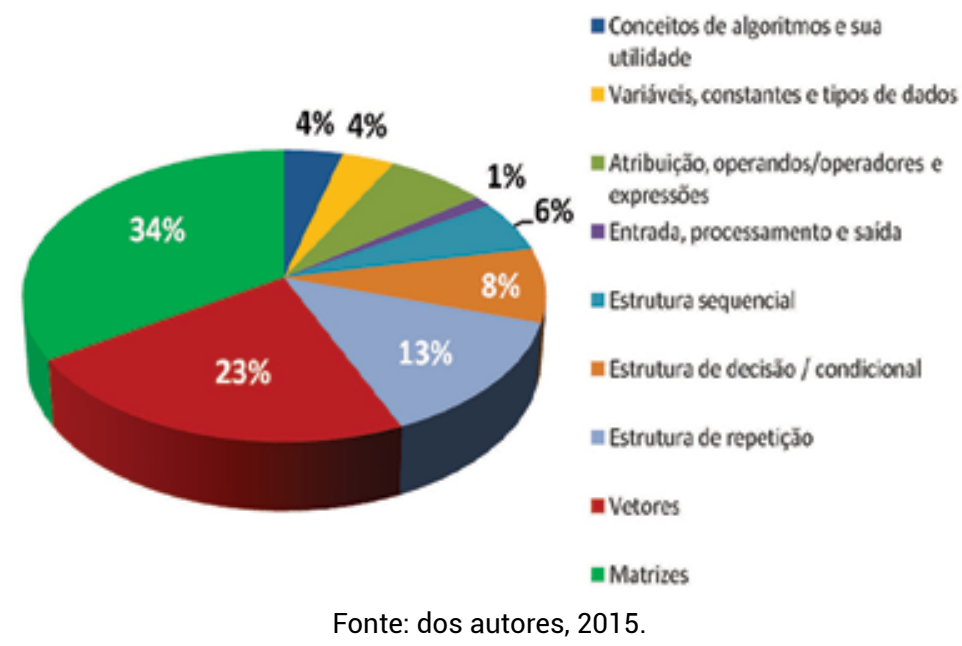

\section{CONCLUSÕES}

A disciplina de Algoritmos é essencial na formação dos discentes dos cursos da área de Computação. Ao longo dos tempos, as experiências em sala de aula e a observação dos altos índices de reprovação motivaram a necessidade de levantamento de informações para que, de forma sistêmica, fosse possível o mapeamento das possíveis causas dos problemas no processo de ensino-aprendizagem.

O instrumento de pesquisa direcionou de forma clara que, dentro do contexto das Unidades de Aprendizagem, existem pontos que devem ser tratados de maneira diferenciada, fugindo do padrão atual, para que se possa aumentar as perspectivas de melhoria no processo de aprendizagem dos alunos.

Um fato observado é que a maior parte dos alunos são egressos do ensino médio regular, que não tiveram base técnica de lógica ou algoritmos, o que contribui de forma direta para o aumento da dificuldade de aprendizado. Os alunos oriundos do segundo grau que fizeram o curso Técnico em Informática sempre apresentam maior facilidade nos conteúdos, o que direciona os índices de aprovação.

Com relação aos conteúdos, a base conceitual inicial não causa grandes dificuldades para os discentes e a organização do pensamento até começa a ser desenvolvida nas primeiras etapas que envolvem praticamente entrada, processamento e saída. No entanto, quando as estruturas de fluxo de algoritmos (sequencial, decisão, repetição) começam a ser apresentadas, os problemas de aprendizagem aparecem de forma clara, culminando com as maiores barreiras, que são as estruturas de dados homogêneas (vetores e matrizes).

Este estudo vem ao encontro das observações registradas de muitos docentes da área de Computação que ministram a disciplina básica de Algoritmos, normalmente, no primeiro ano do curso. É importante que medidas de correção possam ser desenvolvidas para que seja minimizado esse grande problema, que afeta não somente os alunos da pesquisa, mas grande parte dos discentes de todos os cursos de Computação do país.

As propostas de trabalhos futuros são direcionadas para pesquisa e desenvolvimento de metodologias de ensino focadas nas unidades que mais causam dificuldades para os alunos. 0 objetivo é, dentro do contexto, propor novas soluções com base no melhor aproveitamento. 
Dificuldades no processo de aprendizagem de Algoritmos: uma análise dos resultados na disciplina de AL 1 do Curso de Sistemas de Informação da FAETERJ - Campus Paracambi

\section{REFERÊNCIAS}

BALANCHO, M. J. S.; COELHO, F. M. Motivar os Alunos, Criatividade na Relação Pedagógica: Conceitos e Práticas. 2. ed. Alfragide: Editora Texto, 1996.

CARBONI, I. F. Lógica de Programação. São Paulo: Pioneira Thomsor Learning, 2003.

CORMEN, T. H. et al. Algoritmos: Teoria e Prática. 2. ed. Rio de Janeiro: Campus, 2002.

FARRER, H. et al. Algoritmos Estruturados. 3. ed. Rio de Janeiro: Livros Técnicos e Científicos Editora S.A., 1999.

FERREIRA, M. A. S. Proposta de uma Metodologia para Ensino-Aprendizagem de Algoritmos. 2005. $88 \mathrm{f}$. Trabalho de Conclusão de Curso (Bacharelado em Informática) - Universidade do Planalto Catarinense, Lages, 2005.

FORBELLONE, A. L. V.; EBERSPACHER, H. F. Lógica de Programação. 3. ed. São Paulo: Makron Books, 2005.

JESUS, A. de; BRITO, G. S. Concepção de Ensino-Aprendizagem de Algoritmos e Programação de Computadores: a Prática Docente. Varia Scientia, v. 09, n. 16, p. 149-158, 2009.

KOLIVER, C.; DORNELES, R.V.; CASA, M. E. Das (Muitas) Dúvidas e (Poucas) Certezas do Ensino de Algoritmos. In: WORKSHOP SOBRE EDUCAÇÃO EM COMPUTAÇÃO, 24, 2004, Salvador. Anais ... Salvador: UFBA, 2004.

LIBÂNIO, J. C. Didática. São Paulo: Cortez, 1990.

MEDINA, M.; FERTIG, C. Algoritmos e Programação: Teoria e Prática. 2. ed. São Paulo: Novatec, 2006.

MORAES, C. R.; VARELA, S. Motivação do Aluno durante o Processo de Ensino-Aprendizagem. Revista Eletrônica de Educação, Ano I, n. 01, p. 1-15, 2007.

RAABE, A. L. A.; SILVA, J. M. C. da. Um Ambiente para Atendimento às Dificuldades de Aprendizagem de Algoritmos. In: WORKSHOP SOBRE EDUCAÇÃO EM COMPUTAÇÃO, 25, 2005, Canela. Anais ... Canela: UFRGS, 2005.

SOCIEDADE BRASILEIRA DE COMPUTAÇÃO. Currículo de Referência da SBC para Cursos de Graduação em Computação e Informática. 2003.

PACIEVITCH, T. Didática. 2009. Disponível em: <http://www.infoescola.com/pedagogia/didatica/>. Acesso em: 15 mar. 2013. 


\section{APÊNDICE A - QUESTIONÁRIO APLICADO AOS ALUNOS}

IDENTIFICAÇÃO PESSOAL

\begin{tabular}{|c|c|c|}
\hline 01. & Nome: & 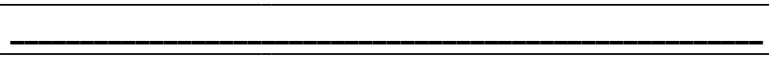 \\
\hline 02. & Sexo: & [] Masculino [] Feminino \\
\hline 03. & Idade: & anos \\
\hline 04. & Período: & \\
\hline 05. & Ensino Medio & $\begin{array}{l}\text { [] Técnico em informática [] Formação de professores } \\
\text { [] Regular [ ] Outros }\end{array}$ \\
\hline 06. & Dependências em AL1: & [] 0 [] 1 [] 2[] 3[] mais de 3 \\
\hline
\end{tabular}

INFORMAÇÕES INICIAIS

\begin{tabular}{ll}
\hline 07. Já havia estudado lógica de programação ou \\
algoritmos em outro lugar?
\end{tabular}

08. de problemas voltados para lógica de

[ ] SIM [ ] NÃO programação?

\section{[] raciocínio lógico}

Quais as suas dificuldades para o entendimento [] capacidade de abstração

09. dos problemas? (podem ser marcados mais de um)

[] leitura e interpretação de textos

[] conhecimentos matemáticos

INFORMAÇÕES SOBRE A DISCIPLINA DE AL1

[] conceitos de algoritmos e sua utilidade

[] variáveis, constantes e tipos de dados

[] atribuição, operandos e expressões

[] entrada, processamento e saída

10. Dentro da disciplina de AL1 qual o conteúdo que [] estrutura sequencial maior causou dificuldades de aprendizado? [ ] estrutura condicional (SE...SENÃO / CASO)

[ ] estrutura de repetição (REPITA, ENQUANTO,

PARA)

[] Vetores

[] Matrizes

11. Você acredita que se dedicou o suficiente para a disciplina de AL1 durante o curso da mesma?

[] SIM [ ] NÃO

12. Você possui(ía) o hábito de fazer algoritmos de forma sistemática todos os dias?

[] SIM [ ] NÃO

[] 01 por dia

[] mais de 1 por dia

13. Qual a quantidade de algoritmos e com que frequência fazia algoritmos fora de sala de aula

[] menos de 3 por semana

[] somente durante as aulas

[] quase não fazia

[] indiferente

14. Das disciplinas cursadas até o momento, AL1 se [] a mais difícil enquadraria em qual opção?

[ ] entre as duas mais difíceis

[] entre as três mais difíceis 